\title{
Acute Chest Syndrome: Can a Chest Radiograph Predict the Course Severity of the Disease?
}

\author{
Arie Franco ${ }^{\mathrm{a}, \mathrm{c}}$, Kathleen Tarrant McKie ${ }^{\mathrm{b}}$, Patrick Ryan Henderson ${ }^{\mathrm{a}}$, Kristopher Neal Lewis ${ }^{\mathrm{a}}$, \\ Roger Anthony Vega ${ }^{b}$
}

\begin{abstract}
Background: The severity and predictability of acute chest syndrome (ACS) varies among patients with sickle cell disease. In our study we analyze whether the pathology identified in the first chest radiograph predict the course severity of ACS.

Methods: We retrospectively reviewed the clinical records and radiographs of 79 episodes of acute chest syndrome in 63 patients with sickle cell disease. We established three categories of severity based on the following parameters: length of admission more than three days, presence of hypoxia, intensive care unit stay, and need for intubation. Two radiologists independently reviewed the first chest radiograph performed on the day of admission. The radiologist graded the degree of pathology and assigned it to one of four levels. Level 1 was defined as complete whiteout of the lungs or consolidation in any lobe with existence of pleural effusion; Level 2 consolidation in 4 lobes; Level consolidation in 3 lobes and level 4 as consolidation in 2 lobes.
\end{abstract}

Results: We calculated the sensitivity, specificity and receiver-operating curve in all three severity categories. In all categories the area under the curve of the receiver-operating curve was above 0.5 , archiving statistical significance.

Conclusions: Patients presenting with multiple lobe involvement

Manuscript accepted for publication January 19, 2012

${ }^{\mathrm{a}}$ Georgia Health Sciences University, Department of Radiology, 1120 15th Street, Augusta, GA 30912, USA

${ }^{\mathrm{b}}$ Georgia Health Sciences University, Department of Pediatrics, 1120 15th Street, Augusta, GA 30912, USA

cCorresponding author: Arie Franco. Email: afranco@georgiahealth.edu

doi:10.4021/ijcp4w in the first chest radiograph had a worse clinical course. These patients might benefit from more aggressive therapy. Our study suggests that predicting the disease severity based on admission chest radiograph may be a useful tool allowing early intervention in the disease course to prevent clinical deterioration and shorten length of stay.

Keywords: Acute chest syndrome; Sickle cell disease; Chest

\section{Introduction}

The sickle cell gene is present in approximately $8 \%$ of the African American (AA) population in the United States and the incidence of sickle cell disease is 1 in 625 in AA in the USA. The most common forms of the disease is the homozygous sickle cell anemia $(\mathrm{S} / \mathrm{S})$, but double heterogeneous states such as sickle-thalassemia (S- $\beta^{0}$ Thalassemia) can be equally affected. The genetic etiology of this disease is substitution of the amino acid valine with glutamic acid in the sixth position of the $\beta$-chain of the hemoglobin tetramer. The amino acid substitution causes the red blood cell to sickle and elongate upon deoxygenation. This leads to microvascular obstruction, tissue ischemia, and infarction. These patients suffer of multi-organ damage including the thoracic organs. Death from homozygous sickle cell anemia usually occurs in a bimodal distribution between birth and 5 years of age with a second peak during the 20 to 25 year age range [1-4]. Acute chest syndrome is the leading cause of hospitalization and death among patients with sickle cell disease.

Acute chest syndrome is an acute pulmonary disease that occurs in patients with sickle cell disease. Acute chest syndrome is defined as a new infiltrate on chest radiograph in conjunction with one other new symptom: chest pain, cough, wheezing, tachypnea, and/or fever $[5,6]$. The term ACS was first suggested in 1979 by Charache et al [7] and reflects the unique nature of the disease. The chest radiograph remains the cornerstone diagnostic test for ACS syndrome. The chest radiograph reveals a new infiltrate most often involving the lower lobe, but any lobe can be affected. Multilobar involvement is also common, and effusions can be present [8-10]. 


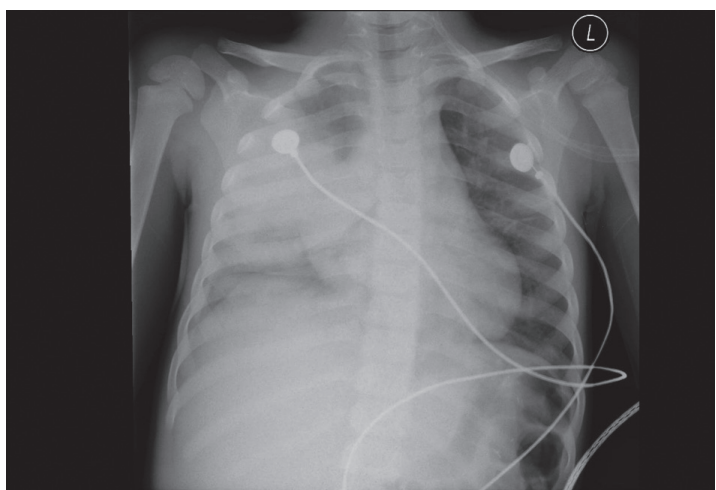

Figure 1. 42-month-old male with acute chest syndrome. Frontal view of the chest reveal consolidations in the right lower lobe, right upper lobe, and left lower lobe. Right-sided pleural effusion is present. Patient was hospitalized for 14 days in the intensive care unit. During hospitalization patient was intubated and his category of clinical course severity was 1 . There are consolidations in three lobes with rightsided pleural effusion and his cut-off level in interpreting the chest radiograph was classified at level 1.

The severity of ACS varies among patients with sickle cell disease. The course of the disease is unpredictable upon admission. Many patients with ACS deteriorate after admission and require treatment in the intensive care unit. Patients suspected of having ACS had a chest radiograph upon admission. In our study we analyzed whether the chest radio-

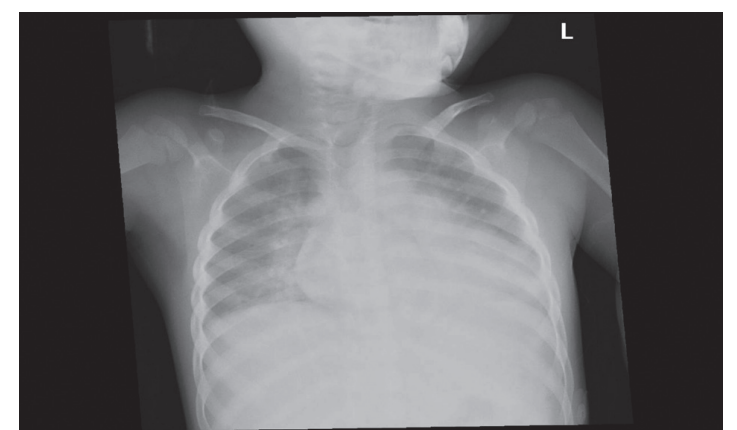

Figure 2. 54-month-old female with acute chest syndrome. Frontal view of the chest reveal consolidation in the right upper lobe, right lower lobe, left lower lobe, and left upper lobe. Patient was hospitalized for 14 days and was intubated in the intensive care unit. His category of clinical severity was 1 and the cut-off level in interpreting the chest radiograph was 2 .

graph could predict the course severity of the disease. Ability to predict severity may affect clinical management, shorten hospital stay, and be more cost-effective.

\section{Methods}

Following approval from the institutional review board, we performed a retrospective review of the medical records and chest radiographs of 63 patients with homozygous sickle
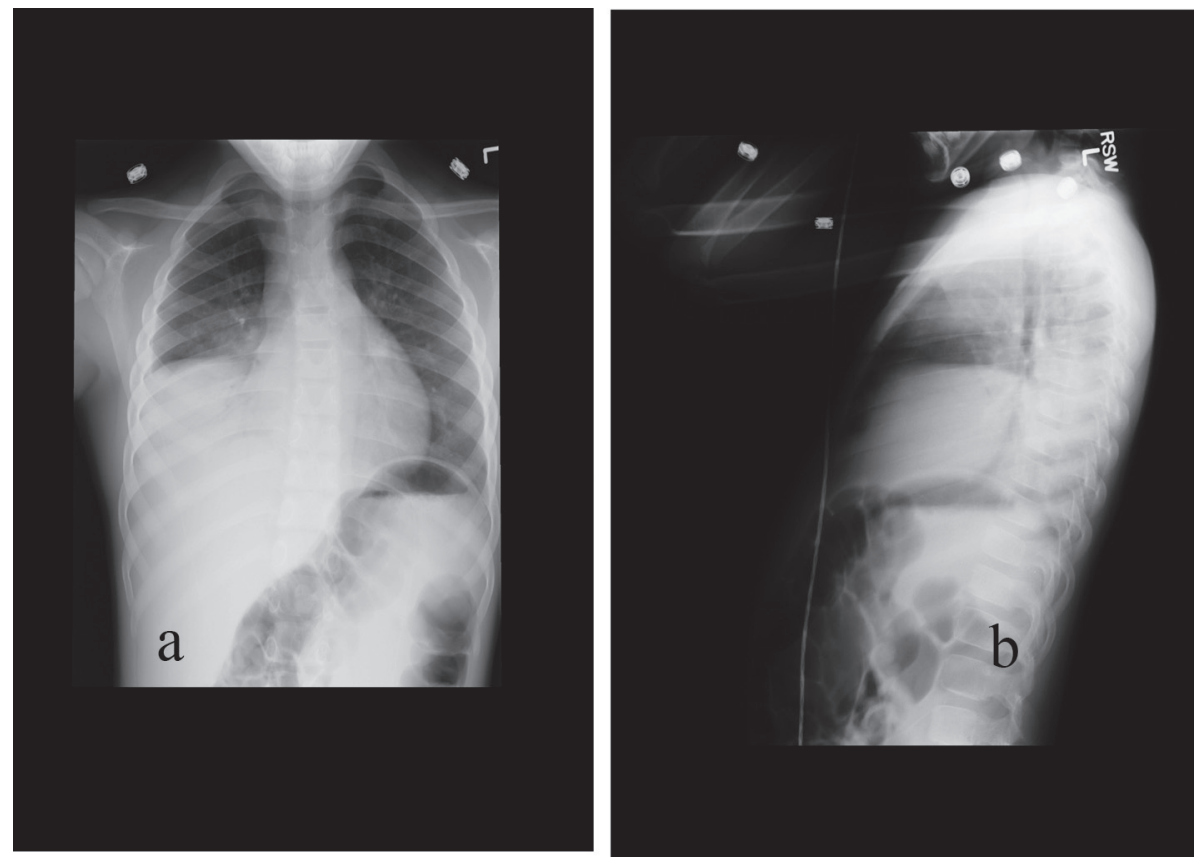

Figure 3. 77-month-old male with acute chest syndrome. Frontal (a) and lateral (b) views of the chest reveal consolidations in the right lower lobe, right middle lobe, and in part of the right upper lobe. Patient was hospitalized for 4 days and had episodes of hypoxia. He was classified in category 2 of severity of clinical course. His cut-off level in interpreting of the chest radiograph was classified at level 3. 

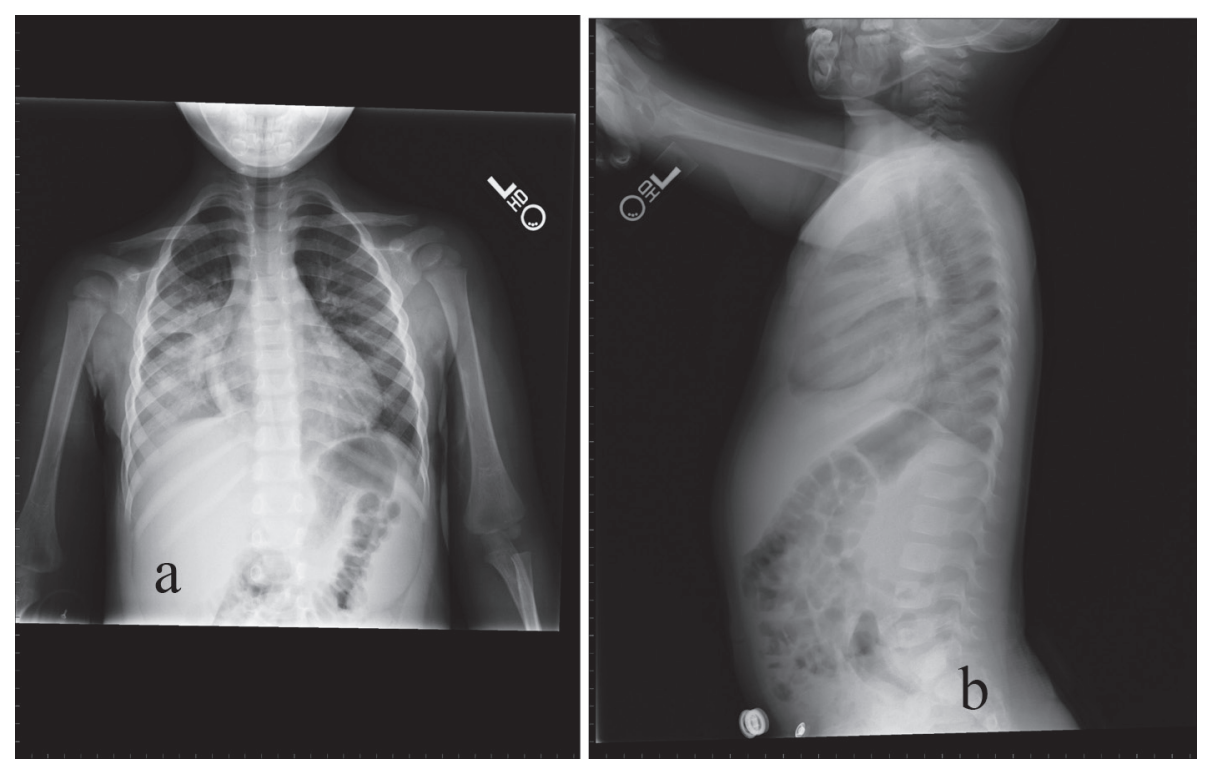

Figure 4. 32-month-old male with acute chest syndrome. Frontal (a) and lateral (b) radiographs of the chest reveal consolidations in the right lower lobe and in the right upper lobe. The patient was hospitalized for 6 days in the intensive care unit and was classified in the clinical course severity as category 3 . As there is consolidation in only two lobes the cut-off level for interpreting the chest radiograph was classified at level 4.

Table 1. Distribution of the 17 Patients, Being Below 3 Years Old

\begin{tabular}{lll}
\hline & Test + & Test - \\
\hline Severity + & 5 & 0 \\
Severity - & 7 & 5 \\
\hline
\end{tabular}

test+ means multilobar infiltrates; test- means single lobe infiltrate; severity+ means severe clinical course as defined in category 1 ; severity- means patients who did not meet the criteria for severe course of category 1.

Table 3. Distribution of the 38 Patients, Being Above Years Old

\begin{tabular}{lll}
\hline & Test + & Test - \\
\hline Severity + & 15 & 3 \\
Severity - & 5 & 15 \\
\hline
\end{tabular}

test+ means multilobar infiltrates; test- means single lobe infiltrate; severity+ means severe clinical course as defined in category 1 ; severity- means patients who did not meet the criteria for severe course of category 1 .

cell disease who were admitted to our institution with ACS. Some of the patients had more than one admission for ACS. For patients with multiple episodes, the interval between
Table 2. Distribution of the 24 Patients, Being Between 3 and 6 Years Old

\begin{tabular}{lll}
\hline & Test + & Test - \\
\hline Severity + & 7 & 2 \\
Severity - & 9 & 6 \\
\hline
\end{tabular}

test+ means multilobar infiltrates; test- means single lobe infiltrate; severity+ means severe clinical course as defined in category 1 ; severity- means patients who did not meet the criteria for severe course of category 1.

Table 4. Distribution of Our Entire Group of 79 Patients at all Ages in the Category 1 of Severity

\begin{tabular}{lll}
\hline & Test + & Test - \\
\hline Severity + & 27 & 5 \\
Severity - & 21 & 26 \\
\hline
\end{tabular}

test+ means multilobar infiltrates; test- means single lobe infiltrate; severity+ means severe clinical course as defined in category 1 ; severity- means patients who did not meet the criteria for severe course of category 1.

episodes studied was at least six months. Two radiologists independently reviewed the first chest radiograph performed 


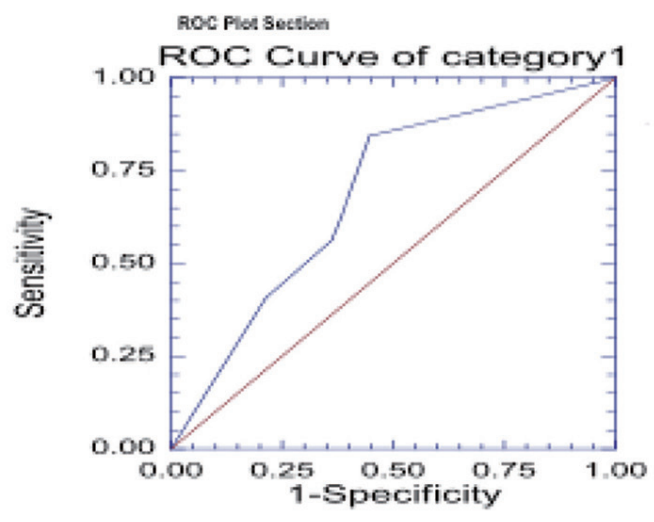

\author{
Sensitivity: $84 \%$ \\ Specificity: $55 \%$ \\ Likelihood ratio: 1.88 \\ Positive predictive value: 0.56 \\ Negative predictive value: 083
}

\begin{tabular}{|c|c|c|c|c|}
\hline Binormal & AUC's & Lower $95.0 \%$ & Upper $95.0 \%$ & Prevalence \\
\hline $\begin{array}{l}\text { Estimate of } \\
\text { AUC }\end{array}$ & $\begin{array}{l}\text { Standard } \\
\text { Error }\end{array}$ & $\begin{array}{l}\text { Confidence } \\
\text { Limit }\end{array}$ & $\begin{array}{l}\text { Confidence } \\
\text { Limit }\end{array}$ & $\begin{array}{c}\text { of } \\
\text { category1 }\end{array}$ \\
\hline 0.67028 & 0.05961 & 0.53642 & 0.77124 & 0.40506 \\
\hline
\end{tabular}

Figure 5. Receiver operating curve in category 1 of severity. The curve was generated with the different threshold levels as defined in the methods section in the entire group of 79 patients. The estimated area under the curve is 0.67028 (above 0.5 ) confirming that chest radiograph is a valid test to predict severe outcome as defined in category 1.

Table 5. Distribution of the 17 Patients, Being Below 3 Years Old

\begin{tabular}{lll}
\hline & Test + & Test - \\
\hline Severity + & 6 & 1 \\
Severity - & 6 & 4 \\
\hline
\end{tabular}

test+ means multilobar infiltrates; test- means single lobe infiltrate; severity+ means severe clinical course as defined in category 2 ; severity- means patients who did not meet the criteria for severe course of category 2 .

Table 7. Distribution of the 38 Patients, Being Above Years Old

\begin{tabular}{lll}
\hline & Test + & Test - \\
\hline Severity + & 16 & 6 \\
Severity - & 4 & 12 \\
\hline
\end{tabular}

test+ means multilobar infiltrates; test- means single lobe infiltrate; severity+ means severe clinical course as defined in category 2; severity- means patients who did not meet the criteria for severe course of category 2 .
Table 6. Distribution of the 24 Patients, Being Between 3 and 6 Years Old

\begin{tabular}{lll}
\hline & Test + & Test - \\
\hline Severity + & 13 & 6 \\
Severity - & 3 & 2 \\
\hline
\end{tabular}

test+ means multilobar infiltrates; test- means single lobe infiltrate; severity+ means severe clinical course as defined in category 2 ; severity- means patients who did not meet the criteria for severe course of category 2 .

Table 8. Distribution of Our Entire Group of 79 Patients at all Ages in the Category 2 of Severity

\begin{tabular}{lll}
\hline & Test + & Test - \\
\cline { 1 - 2 } Severity + & 35 & 13 \\
Severity - & 13 & 18 \\
\hline test+ means multilobar infiltrates; test- means \\
single lobe infiltrate; severity+ means severe \\
clinical course as defined in category 2; se- \\
verity- means patients who did not meet the \\
criteria for severe course of category 2.
\end{tabular}

on admission day.

The clinical severity of the disease course is subjective, and assessment of severity may differ among care providers. In order to standardize the clinical severity of ACS, we established three categories based on the following parameters: length of admission more than three days, presence of hypoxia, intensive care unit stay, and need for intubation. 


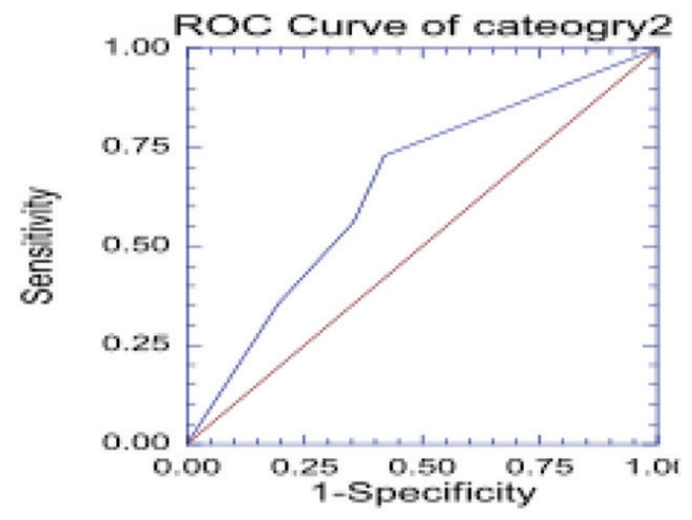

Sensitivity: $72 \%$

Specificity: $58 \%$

Likelihood ratio: 1.73

Positive predictive value: 0.72

Negative predictive value: 0.58

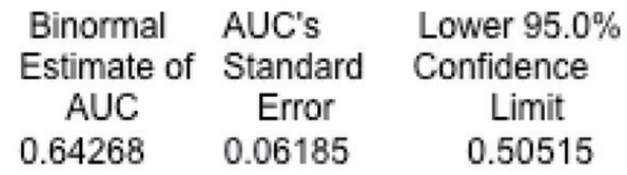

Figure 6. Receiver operating curve in category 2 of severity. The curve was generated with the different threshold levels as defined in the methods section in the entire group of 79 patients. The estimated area under the curve is 0.64268 (above 0.5 ) confirming that chest radiograph is a valid test to predict severe outcome as defined in category 2.
We classified Category 1 as the most severe course of the disease. It was defined by the presence of either intensive care unit stay and/or intubation. Category 2 , intermediate severity, was defined as the presence of hypoxia in addition to hospital stay more than three days. Category 3, the least severe course was defined as the presence of hypoxia or hospital stay more than three days. All our patients were classified in one of these categories.

We examined the admission chest radiograph of each encounter for correlation with disease severity based on the categories described above. Further radiographs after the day of admission were not studied.

We assigned each radiograph to one of these four cut-off levels. Level 1 was defined as complete whiteout of the lungs or consolidation in any lobe with existence of pleural effusion (Fig.1). Level 2 was defined as consolidation in 4 lobes (Fig. 2). Level 3 was defined as consolidation in 3 lobes (Fig. 3 ) and level 4 as consolidation in 2 lobes (Fig. 4).

In order to identify whether findings on admission chest radiograph were more predictive of severity in one age group than another, we divided our patients into three groups according to age. The groups were as follows: Group 1 - below 3 years of age (17 episodes of ACS; 14 patients; mean age: 23.7 months); Group 2 - between 3 and 6 years of age ( 24 episodes of ACS; 21 patients; mean age: 51.6 months); and Group 3 - above 6 years of age (38 episodes; 28 patients; mean: 106.6 months; max: 206 months).

We evaluated every severity category and age groups for sensitivities, specificities and the receiver operating curve to establish whether the first chest radiograph predicted clinical severity.

\section{Results}

The sensitivity of the chest radiograph to predict severity classified as category 1 for the age group below 3 years was $100 \%$ and the specificity was $42 \%$ (Table 1 ).

The sensitivity of the chest radiograph to predict severity classified as category 1 for the age group between 3 and 6 years was $78 \%$ and the specificity was $40 \%$ (Table 2).

The sensitivity of the chest radiograph to predict severity classified as category 1 for the age group above 3 years was $83 \%$ and the specificity was $75 \%$ (Table 3 ).

The total sensitivity of the severity category 1 was $84 \%$ and the specificity $55 \%$ (Table 4 ). Receiver operating curve (Fig. 5) for the entire 79 cases in severity category 1 reveals area of 0.67028 under the curve; sensitivity is $84 \%$ specificity $55 \%$.

The sensitivity of the chest radiograph to predict severity classified as category 2 for the age group below 3 years was $85 \%$ and the specificity was $66 \%$ (Table 5 ).

The sensitivity of the chest radiograph to predict severity classified as category 2 for the age group between 3 and 6 years was $68 \%$ and the specificity was $66 \%$ (Table 6 ).

The sensitivity of the chest radiograph to predict severity classified as category 2 for the age group above 3 years was $72 \%$ and the specificity was $74 \%$ (Table 7 ).

The total sensitivity of the severity category 2 was $72 \%$ and the specificity $58 \%$ (Table 8 ). Receiver operating curve 
Table 9. Distribution of the 17 Patients, Being Below 3 Years Old

\begin{tabular}{lll}
\hline & Test + & Test - \\
\hline Severity + & 10 & 1 \\
Severity - & 2 & 4 \\
\hline
\end{tabular}

test+ means multilobar infiltrates; test- means single lobe infiltrate; severity+ means severe clinical course as defined in category 3 ; severity- means patients who did not meet the criteria for severe course of category 3 .

Table 11. Distribution of the 38 Patients, Being Above Years Old

\begin{tabular}{lll}
\hline & Test + & Test - \\
\hline Severity + & 19 & 10 \\
Severity - & 1 & 8 \\
\hline
\end{tabular}

test+ means multilobar infiltrates; test- means single lobe infiltrate; severity+ means severe clinical course as defined in category 3 ; severity- means patients who did not meet the criteria for severe course of category 3 .

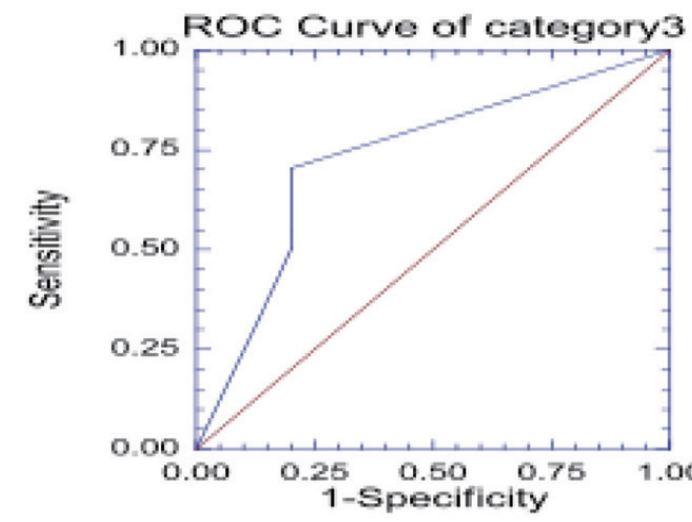

$\begin{array}{lcc}\text { Binormal } & \text { AUC's } & \text { Lower } 95.0 \% \\ \text { Estimate of } & \begin{array}{c}\text { Standard } \\ \text { Confidence } \\ \text { AUC }\end{array} & \begin{array}{c}\text { Error } \\ \text { Limit }\end{array} \\ 0.72195 & 0.06873 & 0.55829\end{array}$

Figure 7. Receiver operating curve in category 3 of severity. The curve was generated with the different threshold levels as defined in the methods section in the entire group of 79 patients. The estimated area under the curve is 0.72195 (above 0.5 ) confirming that chest radiograph is a valid test to predict severe outcome as defined in category 3.

(Fig. 6) for the entire 79 cases in severity category 2 reveals area of 0.64268 beneath the curve.

The sensitivity of the chest radiograph to predict severity classified as category 3 for the age group below 3 years
Table 10. Distribution of the 24 Patients, Being Between 3 and 6 Years Old

\begin{tabular}{lll}
\hline & Test + & Test - \\
\hline Severity + & 16 & 7 \\
Severity - & 0 & 1 \\
\hline
\end{tabular}

test+ means multilobar infiltrates; test- means single lobe infiltrate; severity+ means severe clinical course as defined in category 3 ; severity- means patients who did not meet the criteria for severe course of category 3.

Table 12. Distribution of Our Entire Group of 79 Patients at all Ages in the Category 3 of Severity

\begin{tabular}{lll}
\hline & Test + & Test - \\
\hline Severity + & 45 & 18 \\
Severity - & 3 & 13 \\
\hline
\end{tabular}

test+ means multilobar infiltrates; test- means single lobe infiltrate; severity+ means severe clinical course as defined in category 3 ; severity- means patients who did not meet the criteria for severe course of category 3 .

Sensitivity: $71 \%$

Specificity: $81 \%$

Likelihood ratio: 3.51

Positive predictive value: 0.93

Negative predictive value: 0.38 was $91 \%$ and the specificity was $67 \%$ (Table 9 ).

The sensitivity of the chest radiograph to predict severity classified as category 3 for the age group between 3 and 6 years was $69 \%$ and the specificity was $100 \%$ (Table 10 ). 
The sensitivity of the chest radiograph to predict severity classified as category 3 for the age group above 3 years was $65 \%$ and the specificity was $89 \%$ (Table 11 ).

The total sensitivity of the severity category 3 was $71 \%$ and the specificity $81 \%$ (Table 12 ). Receiver operating curve (Fig. 7) for the entire 79 cases in severity category 3 reveals area of 0.72195 beneath the curve.

\section{Discussion}

Our results show that admission chest radiograph is a good predictor for clinical severity of acute chest syndrome. For the severity categories 1 and 2 the sensitivity exceeds specificity, while in the category 3 the specificity exceeds the sensitivity. This may be explained by the less restrictive criteria for severity in category 3 , leading to more false negative results. In all the three categories the ROC curve is above the mean and the area beneath the curve above 0.5 , suggesting that the chest radiograph is a reliable test.

Due to tighter definition for severe course of disease, the prevalence of the severity in category 1 is the lowest. Nevertheless, the sensitivity of the test is the highest. A low number of patients with severe course of disease increases the false positive rate and decreases the specificity. The specificity increases in category 2 and in category 3 with looser definition of the disease severity; however, the sensitivity does not significantly decrease.

We divided each category of severity into three age groups, but we could not make any significant statistical analysis for each group separately, due to the small sample size. Sensitivity and specificity for each age group is specified and is in the reasonable range.

In a previous study [11], $48 \%$ of pediatric chest radiographs of patients with sickle cell disease admitted to the emergency department were normal. Our study included only pediatric patients who met the defined criteria of acute chest syndrome with an infiltrate in the chest radiograph and who were hospitalized.

We did not perform statistical analysis of other structures in the chest radiograph, since they are not part of the defined acute chest syndrome. However, we did not observe any osseous changes in the majority of our patients, such as $\mathrm{H}$-shaped vertebrae or vascular necrosis of the femoral head. This conforms to a study [12] that suggested that these findings usually increase with age. These findings were found in an adult cohort to be a result of the more prolonged exposure to anemia and skeletal vascular occlusion.

In the majority of our patients there was a lower lobe infiltrate. This also conforms to a study [13] that documents lower lung zone air-space opacity in $64.8 \%$ of the studied patients. Although cardiomegaly was noticed in some of our patients, it was not analyzed. The lung opacity in few of our patients could have been attributable to congestive heart fail- ure; however, this does not change the diagnosis of acute chest syndrome. Both adult and pediatric patients with sickle cell disease develop cardiomegaly at a relatively early age. Cardiomegaly and pneumonia are the most common findings at autopsy in sickle cell patients [14].

The etiology of acute chest syndrome is unclear. In a previous study [15], no identifiable cause was found in $87 \%$ of episodes. An identifiable etiology was found in only $13 \%$ of patients, usually bacterial pneumonia [15]. Etiology may be due to fat embolism, infection, intravascular thrombosis, hypoventilation, over hydration, and an altered environment for adequate chemical oxidation and reduction [16].

The management of Acute Chest Syndrome encompasses a multimodal approach. The potential for infection should be addressed with broad-spectrum antibiotics including mycoplasma coverage. Any degree of dehydration should be addressed by replacing the fluid deficit giving maintenance fluid plus insensible loses. The tachypnea, hypoxia, and anemia should be addressed by bronchodilators, oxygen therapy, and early transfusion. Some patients may require respiratory support with non-invasive mechanical ventilation or intubation.

Our study showed that patients presenting with multiple lobe involvement had a worse course. These patients might benefit from more aggressive intervention that may include consultation with the intensive care unit and exchange transfusions. Our study suggests that prediction of disease severity based on admission chest radiograph may be a useful tool allowing intervention early in the disease course to prevent clinical deterioration and shorten length of stay.

\section{References}

1. Leong CS, Stark P. Thoracic manifestations of sickle cell disease. J Thorac Imaging. 1998;13(2):128-134.

2. Vichinsky EP. Comprehensive care in sickle cell disease: its impact on morbidity and mortality. Semin Hematol. 1991;28(3):220-226.

3. Castro O, Brambilla DJ, Thorington B, Reindorf CA, Scott RB, Gillette P, Vera JC, et al. The acute chest syndrome in sickle cell disease: incidence and risk factors. The Cooperative Study of Sickle Cell Disease. Blood. 1994;84(2):643-649.

4. Platt OS, Brambilla DJ, Rosse WF, Milner PF, Castro O, Steinberg MH, Klug PP. Mortality in sickle cell disease. Life expectancy and risk factors for early death. N Engl J Med. 1994;330(23):1639-1644.

5. Platt OS. The acute chest syndrome of sickle cell disease. N Engl J Med. 2000;342(25):1904-1907.

6. Bernard AW, Yasin, Z, Venkat, A. Acute chest syndrome of sickle cell disease. Hospital Physician 2007; 44: 1523.

7. Charache S, Scott JC, Charache P. "Acute chest syndrome" in adults with sickle cell anemia. Microbi- 
ology, treatment, and prevention. Arch Intern Med. 1979;139(1):67-69.

8. Vichinsky EP, Styles LA, Colangelo LH, Wright EC, Castro O, Nickerson B. Acute chest syndrome in sickle cell disease: clinical presentation and course. Cooperative Study of Sickle Cell Disease. Blood. 1997;89(5):17871792.

9. Vichinsky EP, Neumayr LD, Earles AN, Williams R, Lennette ET, Dean D, Nickerson B, et al. Causes and outcomes of the acute chest syndrome in sickle cell disease. National Acute Chest Syndrome Study Group. N Engl J Med. 2000;342(25):1855-1865.

10. Sprinkle RH, Cole T, Smith S, Buchanan GR. Acute chest syndrome in children with sickle cell disease. A retrospective analysis of 100 hospitalized cases. Am J Pediatr Hematol Oncol. 1986;8(2):105-110.

11. Miller JA, Hinrichs CR. Sickle cell crisis in the adult: chest radiographic findings and comparison with pediat- ric sickle cell disease. J Natl Med Assoc. 2001;93(2):5863.

12. Riggs W, Jr., Rockett JF. Roentgen chest findings in childhood sickle cell anemia. A new vertebral body finding. Am J Roentgenol Radium Ther Nucl Med. 1968;104(4):838-845.

13. Wong KT, Antonio GE, Hui DS, Lee N, Yuen EH, Wu A, Leung CB, et al. Severe acute respiratory syndrome: radiographic appearances and pattern of progression in 138 patients. Radiology. 2003;228(2):401-406.

14. Smith JA. Cardiopulmonary manifestations of sickle cell disease in childhood. Semin Roentgenol. 1987;22(3):160-167.

15. Martin L, Buonomo C. Acute chest syndrome of sickle cell disease: radiographic and clinical analysis of 70 cases. Pediatr Radiol. 1997;27(8):637-641.

16. Knight J, Murphy TM, Browning I. The lung in sickle cell disease. Pediatr Pulmonol. 1999;28(3):205-216. 